\title{
The perception of shrinking in apparent motion
}

\author{
MAURICE HERSHENSON \\ Brandeis University, Waltham, Massachusetts
}

\begin{abstract}
Apparent motion was produced using two triangular patterns of different sizes, each exposed for $100 \mathrm{msec}$, with a 50 -msec interstimulus interval and 200 -msec recycle interval. The triangles were aligned either on center or on the midpoints of the bases. In Experiment 1, filled, outline, and three-dot triangles were viewed over four backgrounds: a blank illuminated field, and texture gradients constructed from horizontal lines, perspective lines, or a combination of these (full texture). In Experiment 2, outline and dot triangles were presented in one of three orientations: base down, base right, and base up over a blank background. Subjects made two forced-choice responses: apparent size was categorized as shrinking or not shrinking, and apparent motion was categorized as motion in depth or motion in a fixed frontal plane. The type of alignment was the major determiner of responses. When the midpoints of the base were aligned, the predominant response described a shrinking object in a fixed position in depth. When the centers were aligned, the predominant response described an object of constant size moving in depth.
\end{abstract}

To say that an object "shrinks" means that it gets smaller but retains its shape. The illusion of shrinking has been produced in cartoon, science fiction, and fantasy films, leaving no doubt that a two-dimensional stimulus can appear to shrink in size. Nevertheless, the mechanism underlying this illusion is not understood. Clearly the perception of shrinking is not produced simply by a decreasing proximal stimulus size. Such a stimulus typically produces the perception of motion in depth, a proximal-perceptual relationship that is well established (Gibson, 1950; Hershenson, 1982, 1991; Ittelson, 1951; Johansson, 1964; Schiff, 1965). Therefore, the stimulus that produces the perception of shrinking must differ in some way from the stimulus that produces the perception of motion in depth. This report describes two experiments that examined possible sources of the difference: (1) the proximal motion pattern of the shrinking object, (2) its height in the picture plane, and (3) the background on which it appeared.

Normally, three-dimensional motions of rigid objects project as proximal size change in two dimensions; this is, in turn, perceived as rigid objects moving in threedimensions. Real shrinking objects (such as deflating balloons) also project as two-dimensional proximal size change. However, their appearance is illusory-they appear to be rigid objects moving in depth rather than stationary objects that are changing in size (Ittelson, 1951). Movies provide a clue to the difference in the proximal patterns for objects that appear to shrink. Typically, a shrinking object appears to retain its perceived position

The results of Experiment 1 were reported at the meeting of the Psychonomic Society, San Francisco, November 1991. I thank Ruth D. Edelstein, Eric L. Weinstock, Miriam Linver, and Heidi Factor for collecting the data. I thank Dennis R. Proffitt for suggesting Experiment 2. Address correspondence to $M$. Hershenson, Department of Psychology, Brandeis University, Waltham, MA 02254-9110. in depth. The size change appears to be directed toward a point on one edge of the object, an edge that appears to anchor its position in depth. The proximal representation of this edge expands and contracts in only one dimension. It is possible, then, that perceived shrinking results when one side of a proximal motion pattern changes in size in only one dimension.

This difference is illustrated in Figure 1 for two contracting (converging) triangular proximal motion patterns. Pattern a shows a triangle that is contracting toward its midpoint. The vertices move toward the common central point. The sides decrease in linear size and also change position in the picture plane-the sides move diagonally and the base moves upward. Thus, all three sides move in two dimensions in the picture plane. Pattern b shows a triangle that is contracting about the midpoint of one side (the base). The vertices of this triangle converge toward the midpoint of the base. This pattern differs from Pattern a primarily in the motion of the base: it decreases in size along the axis containing the base but it does not change position in the picture plane. Thus, all sides move in two dimensions in Pattern a, but the base changes in only one dimension in Pattern b. Similar relationships hold for divergence (expansion) patterns.

It is also possible that the dimensional difference in the motion patterns is necessary but not sufficient to produce the perception of shrinking. A textured ground may be required to provide an anchor for the base or to provide a stable context for the relative motions of the other sides. These possibilities were examined in the first experiment using triangular apparent motion patterns composed of dots, lines, and filled shapes. Each pattern was viewed over four backgrounds: (1) a blank illuminated field, (2) lines drawn in perspective converging toward the horizon, (3) a horizontal-line texture gradient, and (4) a full-texture pattern combining the perspective and horizontal line gradients. 


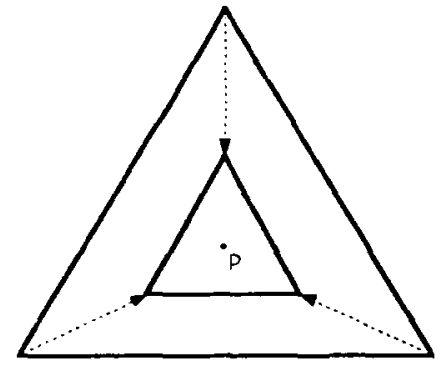

(a) Convergence toward point near center

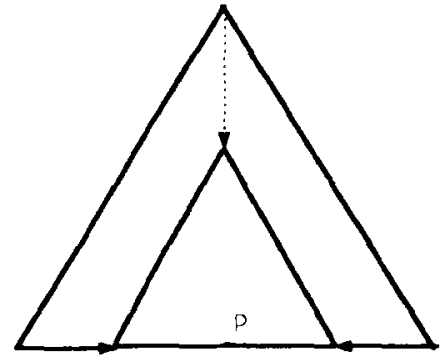

(b) Corivergence toward

point on base

Figure 1. Triangular motion patterns illustrating the difference in relative motions of the base (one side). In Pattern a, the vertices of the triangle move toward a point (P) near the center. The base changes in two dimensions-it decreases in linear size as it moves upward in the picture plane. In Pattern b, the vertices converge toward a point on the base. This side of the triangle changes in one dimension.

\section{EXPERIMENT 1 \\ Backgrounds and Pattern Elements}

\section{Method}

Stimuli. A single stimulus for apparent motion consisted of two cards containing triangles of different sizes, each exposed in one channel of a Scientific Prototype three-channel mirror tachistoscope (Model GB). There were three types of triangles: (1) filled triangles, (2) outline triangles, and (3) triangles with dots placed at the vertices. There were two types of apparent-motion patterns: (1) concentric and (2) collinear base. The vertices of concentric patterns were placed along axes that met at a point near the center of the triangles. These apparent-motion stimuli are similar in position to the pattern illustrated in Figure la. The vertices of collinear base patterns were placed along axes that met at the midpoint of the base of the triangles. These apparent-motion stimuli were similar in position to the pattern illustrated in Figure $1 \mathrm{~b}$. The patterns were presented in base-down (horizontal) orientation, centered in the visual field. They subtended a maximum of $2.5^{\circ}$ of visual angle on a side.

The stimuli were presented against four types of backgrounds exposed in a third channel of the tachistoscope. The blank field was produced by an illuminated white card. The horizontal-line texture consisted of horizontal lines with gradually decreasing intervening spaces from the bottom of the field to the horizon line, which was $1.1^{\circ}$ of visual angle above the center of the field. The perspectiveline texture consisted of lines that ran from the bottom of the field to the horizon line but converged toward a point slightly above the horizon. The full-texture background consisted of a combination of the horizontal and perspective line textures. The rectangular stimulus field measured $4.6^{\circ}$ of visual angle horizontally and $6.4^{\circ}$ of visual angle vertically. It contained a small red fixation cross at the center. The area outside the background field was dark.

Procedure. The stimuli were exposed alternately for $100 \mathrm{msec}$ over a background field that was constantly illuminated. The flashing channels were recycled with a 50-msec interstimulus interval and a 200 -msec recycle interval. The values were selected in pilot testing because they produced strong perceptions of apparent motion. The shorter interval followed the presentation of the larger triangle, making the apparent motion toward the point of convergence the more salient aspect of the perceived motion.

The subjects sat with their heads firmly pressed against a head restraint. Viewing was binocular, but there was no differential information from retinal disparity because the stimulus components were at the same optical distance. (There was no apparent motion in depth from same-sized triangles alternately exposed in the tachistoscope.) Each of the six types of apparent-motion stimuli was presented over each of the four backgrounds for $3 \mathrm{~min}$. The 24 pattern $\times$ motion type $\times$ background combinations were presented in a different predetermined random order for each subject.

The subjects were required to make forced-choice responses, continuously categorizing their perceptions as shrinking or not shrinking and as moving in depth or not. They were instructed to respond to the following questions: (1) Does the object or figure appear to be shrinking and expanding or does it appear to remain the same size? (2) Does the object or figure appear to be moving in depth or does it appear to move only in the frontal plane? The subjects were instructed to respond as soon as possible after stimulus onset and to continue responding during the entire 3-min exposure. They were, however, permitted to remain silent if their perceptions did not change, but were instructed to report a change the moment it took place. The type of change could be described immediately after noting it. The subjects were specifically told that silence would be interpreted as no change in perception since the last description. The instructions were also explained informally. The perceived shrinking in the movie Alice in Wonderland was given as an example of the kind of perception the subject should describe as "shrinking," and the perceived motion in depth seen in the Road Runner cartoons was given as an example of the kind of perception the subject should describe as "motion in depth."

Subjects. The subjects were 9 undergraduate volunteers from an introductory course in psychology. Each had normal or correctedto-normal vision.

\section{Results}

The protocols were continuous records of the subjects' responses describing the perceived object as shrinking or not shrinking and as moving in depth or remaining in the same depth plane. Thus, there were four possible combinations of the two two-choice responses. The data were scored for each response separately and for the combination of responses.

Analyses of variance were computed on two arcsin $x^{1 / 2}$ transforms of proportions separately for shrinking and motion-in-depth reports in each subject $\times$ stimulus pattern $\times$ motion type $\times$ background combination. The only large difference was produced by the position of the point of convergence $[F(1,8)=22.03$ and $31.15, p<.01$, for 
shrinking and motion-in-depth responses, respectively]. None of the other main effects or interactions was significant for the shrink response. For the motion-in-depth response, the only significant interaction was between background and the type of object $[F(6,48)=5.32, p<.01]$. (Note that point of convergence did not interact with any other variable.) The triangular arrays of dots produced the fewest depth responses over the blank background (35\%), the most depth responses over the horizontal-line background $(57 \%)$, and an intermediate number of depth responses over the perspective and full-texture backgrounds (46\% and $51 \%$, respectively). Outline triangles and solid triangles produced similar proportions (between $55 \%$ and $64 \%$ ) for all backgrounds.

Table 1 shows the proportion of shrink and no-shrink responses for the base and center convergence patterns. Perceived shrinkage was reported $80 \%$ of the time when the point of convergence was located on the base of the triangle and $44 \%$ of the time when it was located near the center of the triangle. Table 2 shows the proportion of frontal-plane and motion-in-depth responses for each pattern. Perceived motion in depth was reported $38 \%$ of the time when the convergence point was located on the base of the triangle and $74 \%$ of the time when it was located near the center of the triangle.

The paired responses were evaluated separately in $2 \times$ 2 contingency tables for each of the 24 conditions. Each of the resulting chi-squares was significant $(p<.01)$. Table 3 shows the overall proportions of paired responses for base and center convergence patterns. Perceived shrinking was associated with motion in the frontal plane (i.e., no motion in depth) $62 \%$ of the time when the point of convergence was on the base of the triangle and $26 \%$ of the time when the point of convergence was near the center of the triangle. Perceived motion in depth was associated with a nonshrinking object (i.e., an object of constant size)

Table 1

Proportion of Shrink or No-Shrink Responses for Base and Center Convergence Patterns in Experiment 1

\begin{tabular}{lcc}
\hline & Shrink & No Shrink \\
\hline Base convergence & 80 & 21 \\
Center convergence & 44 & 50 \\
\hline
\end{tabular}

Table 2

Proportion of Frontal-Plane or Depth-Motion Responses for Base and Center Convergence Patterns in Experiment 1

\begin{tabular}{lcc}
\hline & Frontal Plane & Depth Motion \\
\hline Base convergence & 63 & 38 \\
Center convergence & 26 & 74 \\
\hline
\end{tabular}

Table 3

Proportion of Paired Responses for Base and Center Convergence Patterns in Experiment 1

\begin{tabular}{lccccc}
\hline & \multicolumn{2}{c}{ Base Convergence } & & \multicolumn{2}{c}{ Center Convergence } \\
\cline { 2 - 3 } \cline { 5 - 6 } & Shrink & No Shrink & & Shrink & No Shrink \\
\hline Frontal-plane motion & 62 & 1 & & 26 & 0 \\
Motion in depth & 18 & 20 & & 18 & 56 \\
\hline
\end{tabular}

$20 \%$ of the time when the point of convergence was on the base of the triangle and $56 \%$ of the time when the point of convergence was near the center of the triangle. Shrinking and motion in depth were associated $18 \%$ of the time for both convergence patterns, while constancy and frontalplane motion were associated less than $1 \%$ of the time. Assuming that each of the four response combinations was equally likely, it is clear that the position of the point of convergence was a major determiner of the paired response: When the point of convergence was on the base, the predominant response described a shrinking object in the frontal plane; when the point of convergence was near the center, the predominant response described an object of constant size moving in depth.

\section{EXPERIMENT 2 Orientation and Height in Picture Plane}

In Experiment 1, the point of convergence of the apparent motion was either on the base or near the center of the triangle. Because the motion patterns were presented in base-down orientation, the base of the triangle could move in one or in two dimensions, and, consequently, the relative height in the picture plane changed differentially. That is, when the sides converged toward a point on the base, the base remained at the same height, but when the sides converged toward a point near the center, the base moved higher in the frontal plane. Therefore, it is possible that the difference in the responses produced by these two patterns was due to the differential height in the picture plane rather than to the dimensionality of the motion pattern itself.

Experiment 2 addressed this possibility by varying the orientation of the apparent-motion patterns. Three orientations were used: (1) base up, (2) base down, and (3) base right. The base-up pattern reversed the relationship because the upper side (base) moved lower in the picture plane for a convergence point near the center. In the base-right orientation, the center of the side (base) was at the same height in the picture plane as the central point of convergence. Therefore, in this orientation, the sizechange component of the motion of the side (base) did not change its height in the picture plane.

\section{Method}

Stimuli. The stimuli were triangular configurations of dots and lines. Figure 2 shows the concentric (left-hand) and collinear (righthand) patterns in the three orientations: base up, base right, and base down. The stimuli were presented against a blank white background.

Procedure. The procedures and instructions were the same as in Experiment 1. The 12 stimulus pattern $\times$ orientation $\times$ stimulus type combinations were presented in a different predetermined random order for each subject. Eight subjects were assigned randomly to view either outline or dot triangles.

Subjects. The subjects were 16 undergraduates. Each had normal or corrected-to-normal vision.

\section{Results}

The protocols were continuous records of the subjects' responses. The data were scored in the same way as in 
Experiment 1. Analyses of variance were computed on two $\arcsin x^{1 / 2}$ transforms of percent shrinking reports and percent motion-in-depth reports in each stimulus pattern $X$ orientation $\times$ stimulus type combination.

The position of the point of convergence of the apparentmotion pattern produced the only large difference $[F(1,14)=19.83$ and $23.66, p<.01$, for shrinking and motion-in-depth responses, respectively]. Base-up orientation produced the largest proportion of perceived depth responses $(65 \%)$, and base-down orientation produced the smallest proportion $(43 \%)$. Base-right orientation fell in between $(49 \%)[F(2,28)=7.97, p<.01]$. None of the other main effects or interactions was significant.

Table 4 shows the proportion of shrink and no-shrink responses for the base and center convergence patterns. Perceived shrinkage was reported $84 \%$ of the time when the point of convergence was located on a side of the triangle and $38 \%$ of the time when it was located near the center of the triangle. Table 5 shows the proportion of frontal-plane and motion-in-depth responses for each pattern. Perceived motion in depth was reported $30 \%$ of the time when the convergence point was located on a side of the triangle and $70 \%$ of the time when it was located near the center of the triangle.

Table 6 shows the overall contingent relationships between the responses for base and center convergence patterns $\left[\chi^{2}(3)=7,247\right.$ and $8,941, p<.01$, respectively $]$.
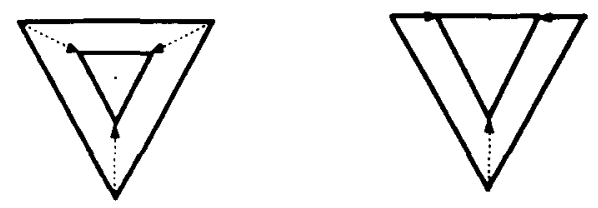

(a) Base up
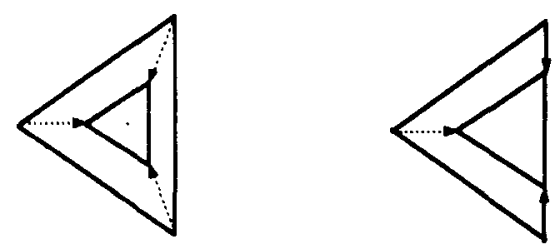

(b) Base right
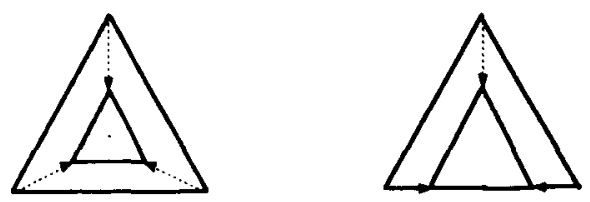

(c) Base down

Figure 2. Concentric (left-side) and collinear (right-side) outline triangles used to produce apparent motion in Experiment 2. Stimuli were presented in one of three orientations: base up, base right, or base down. The arrows indicate primary apparent-motion paths for the vertices.
Table 4

Proportion of Shrink or No-Shrink Responses for Base and Center Convergence Patterns in Experiment 2

\begin{tabular}{lcc}
\hline & Shrink & No Shrink \\
\hline Base convergence & 84 & 17 \\
Center convergence & 38 & 63 \\
\hline
\end{tabular}

Table 5

Proportion of Frontal-Plane or Depth-Motion Responses for Base and Center Convergence Patterns in Experiment 2

\begin{tabular}{lcc}
\hline & Frontal Plane & Depth Motion \\
\hline Base convergence & 69 & 30 \\
Center convergence & 31 & 70 \\
\hline
\end{tabular}

Table 6

Proportion of Paired Responses for Base and Center Convergence Patterns in Experiment 2

\begin{tabular}{lccccc} 
& \multicolumn{2}{c}{ Base Convergence } & & \multicolumn{2}{c}{ Center Convergence } \\
\cline { 2 - 3 } \cline { 5 - 6 } & Shrink & No Shrink & & Shrink & No Shrink \\
\hline Frontal-plane motion & 68 & 1 & & 29 & 2 \\
Motion in depth & 16 & 16 & & 9 & 61 \\
\hline
\end{tabular}

Perceived shrinking was associated with motion in the frontal plane (i.e., no motion in depth) $68 \%$ of the time when the point of convergence was on a side of the triangle and $29 \%$ of the time when the point of convergence was near the center of the triangle. Perceived motion in depth was associated with a nonshrinking object (i.e., constant-sized object) $16 \%$ of the time when the point of convergence was on a side of the triangle and $61 \%$ of the time when the point of convergence was near the center of the triangle. Shrinking and motion in depth were associated $16 \%$ and $9 \%$ of the time for side and center convergence, respectively, while constancy and frontal-plane motion were reported no more than $2 \%$ of the time. Assuming that each of the four response combinations was equally likely, it is clear that the position of the point of convergence was a major determiner of the paired response: When the point of convergence was on a side of the triangle, the predominant response described a shrinking object in the frontal plane; when it was near the center of the triangle, the predominant response described an object of constant size moving in depth.

\section{DISCUSSION}

The experiments demonstrate that a specific proximal pattern-convergence toward a point on a side of the figure-is implicated in the perception of shrinking in a fixed frontal plane. When the qualities were analyzed separately, base-convergence patterns appeared to change in size (shrink and expand), whereas center-convergence patterns appeared to remain constant in size; baseconvergence patterns appeared to remain in a fixed frontal plane, whereas center-convergence patterns appeared to change their position in depth. A consistent response pairing was demonstrated in the contingency analyses: 
When the triangles appeared to shrink, they appeared to be fixed in a single depth plane; when the triangles appeared not to shrink (to remain constant in size), they appeared to move in depth. These perceptions were apparently independent of background, orientation, and height in the picture plane. They cannot be attributed to differential cues to flatness or to prior experience, because these factors were constant across stimuli.

Placing the point of convergence on one side of the figure reduces the proximal change in that side to a single dimension. Many studies have reported the difference in perceptual response to one-dimensional and twodimensional change in the proximal stimulus. Proximal patterns that change in one dimension do not consistently produce a single response. Instead, they result in reports of shape and/or size change as well as constancy, and of rotation and/or translation in depth as well as position constancy. In contrast, patterns that change in two dimensions almost invariably produce reports of rigid object motion in depth (Börjesson \& von Hofsten, 1972, 1973; Johansson, 1950, 1958, 1964; Johansson \& Jansson, 1968; Swanston \& Gogel, 1986; Wallach \& O'Connell, 1953). However, base-convergence patterns were not used in these studies, and, consequently, the specific proximal pattern that consistently produced frontal-plane shrinking was not isolated.

The relationship between perceived size and perceived distance also impacts on our understanding of minimum principle explanations of space perception (Hatfield \& Epstein, 1985). A minimum principle might suggest, for example, that the most parsimonious organization of the input determines the perceptual response. In this view, the center-convergence pattern appears to move in depth and the base-convergence pattern appears to shrink because these perceptions require change in only one dimension: $z$-axis for the former and size for the latter. But this explanation masks the more important feature of the response-the absence of change in perception, in this case constancy of size (rigidity), and of position (frontal-plane motion). The experiments demonstrated these relationships explicitly because the dual forced-choice responses exposed the contingent relationship. In the limiting cases, change in one perceptual attribute was associated with constancy in the other: changing perceived size (shrinking) with constancy of depth-plane position, and changing position in depth with nonchanging size (constancy or rigidity). In this sense, a constancy principle and a minimum principle are the same.

In another context, the demonstration of paradoxical perceptions such as micropsia and the moon illusion has been taken as evidence that perceived size and perceived distance are independent (Haber \& Levin, 1989). The contingent relationship between these perceptual qualities argues against this view. However, the data also suggest that the relationship is not as simple as that proposed in the kinetic version of the size-distance invariance hypothesis (Hershenson, 1982, 1989, 1991, 1992). That hypothesis asserts that a changing solid visual angle produces a constant perceived size and a changing perceived distance. As originally conceived, the description of the proximal stimulus as a changing solid visual angle described a proximal change in area. However, identifying the stimulus for perceived shrinking suggests a modification of the hypothesis because it clearly does not apply to stimuli that appear to shrink. Apparently, kinetic invariance describes the perceptual response to a changing solid visual angle only when all sides of the proximal pattern change in two dimensions.

\section{REFERENCES}

BöREsSON, E., \& vON HoFsTEN, C. (1972). Spatial determinants of depth perception in two-dot motion patterns. Perception \& Psychophysics, 11, 263-268.

Böruesson, E., \& von Horsten, C. (1973). Visual perception of motion in depth: Application of a vector model to three-dot motion patterns. Perception \& Psychophysics, 13, 169-179.

GiBson, J. J. (1950). The perception of the visual world. Boston: Houghton Mifflin.

HABER, R. N., LEVIN, C. A. (1989). The lunacy of moon watching: Some preconditions on explanations of the moon illusion. In $\mathbf{M}$. Hershenson (Ed.), The moon illusion (pp. 299-317). Hillsdale, NJ: Erlbaum.

Hatfield, G., Epstein, W. (1985). The status of the minimum principle in the theoretical analysis of visual perception. Psychological Bulletin, 97, 155-186.

HERSHENSON, M. (1982). Moon illusion and spiral aftereffect: Illusions due to the loom-zoom system? Journal of Experimental Psychology: General, 111, 423-440.

Hershenson, M. (1989). Moon illusion as anomaly, In M. Hershenson (Ed.), The moon illusion (pp. 123-145). Hillsdale, NJ: Erlbaum.

HeRSHENSON, M. (1991, June). Apparent motion of lines and lightpoints: Further evidence for structural constraints. Paper presented at the meeting of the American Psychological Society, Washington, DC.

Hershenson, M. (1992). Size-distance invariance: Kinetic invariance is different from static invariance. Perception \& Psychophysics, 51, 541-548.

ITTELSON, W. H. (1951). Size as a cue to distance: Radial motion. American Journal of Psychology, 64, 188-202.

Johansson, G. (1950). Configurations in event perception. Uppsala, Sweden: Almqvist \& Wiksells.

Johansson, G. (1958). Rigidity, stability, and motion in perceptual space. Acta Psychologica, 14, 359-370.

Johansson, G. (1964). Perception of motion and changing form. Scandinavian Journal of Psychology, 5, 181-208.

Johansson, G., Jansson, G. (1968). Perceived rotary motion from changes in a straight line. Perception \& Psychophysics, 4, 165-170.

SCHIF, W. (1965). Perception of impending collision: A study of visually directed avoidant behavior. Psychological Monographs, 79(Whole No. 604).

Swanston, M. T., \&ogel, W. C. (1986). Perceived size and motion in depth from optical expansion. Perception \& Psychophysics, 39, 309-326.

Wallach, H., \& O'Connell, D. N. (1953). The kinetic depth effect. Journal of Experimental Psychology, 45, 205-217.

(Manuscript received July 26, 1991; revision accepted for publication May 19, 1992.) 